\title{
Carbon nanotubes catalysis in liquid-phase aerobic oxidation of hydrocarbons: Influence of nanotube impurities
}

\author{
Eldar Zeynalov ${ }^{\mathrm{a}}$, Norman S. Allen ${ }^{\mathrm{b}, *}$, Nazilya Salmanova ${ }^{\mathrm{c}}$, Vladimir Vishnyakov ${ }^{\mathrm{d}}$ \\ ${ }^{a}$ Institute of Catalysis \& Inorganic Chemistry, Azerbaijan National Academy of Sciences, 113 H. Javid Ave., Baku, Azerbaijan \\ ${ }^{\mathrm{b}}$ School of Science and the Environment, Faculty of Science and Engineering, Manchester Metropolitan University, Chester Street, Manchester, M1 5GD, UK \\ ${ }^{c}$ Azerbaijan State Oil and Industry University, Ministry of Education, 16/21 Azadliq Ave., Baku, Azerbaijan \\ ${ }^{\mathrm{d}}$ Institute for Materials Science, University of Huddersfield, Huddersfield, HD1 3DH, UK
}

\section{A R T I CLE INFO}

\section{Keywords:}

Carbon nanotubes

CVD process

Electron affinity

Aerobic oxidation of hydrocarbons

Oxygen absorption

Line breakage

Oxidation inhibition

Chain branching

Catalysis

\begin{abstract}
A B S T R A C T
Pure carbon nanotubes (CNTs) have a high electron affinity and as such are able to actively absorb free radicals. This functional feature of CNTs leads to a linear chain breakage with the formation of inert spin adducts and effective inhibition of the oxidation process. However, there is a clear contradiction in this issue in the literature with the analysis of research results indicating that CNTs exhibit antioxidant activity mainly in polymeric materials, under conditions of diffusional restrictions for oxygen access. While, in the liquid-phase oxidation of hydrocarbons by CNTs (CNTs synthesised by the thermal catalytic pyrolysis of carbon-containing raw materials (CVD-process)), the CNTs enhance catalytic processes. In this study the aerobic liquid phase oxidation of cumene and initiated (by azobisisobutyronitrile) at low-temperature (333 K) in the presence of multi-walled carbon nanotubes (MWCNTs) obtained by thermal catalytic pyrolysis of cyclohexane (catalyst-ferrocene) has been undertaken. Kinetic analysis establishes that the catalysis of the oxidation process is associated with the presence of metal compounds in the structure of the MWCNTs. These metals are residues of metal catalysts remaining in the synthesis and in the process of pyrolysis. The metals are converted, as a rule, into metal carbides and are not easily removable by treatment with mineral acids. Thus, in the presence of metals in the composition of MWCNTs, interfering parallel reactions are observed with two processes running in parallel $\left.-\mathrm{MWCNTs}_{+} \bullet \mathrm{RO}_{2} \bullet\right) \rightarrow$ $\bullet$ MWCNTs-R $\left(\mathrm{RO}_{2}\right)$ and ROOH $+\mathrm{M} @$ MWCNTs $\rightarrow$ RO・ radicals $\left(\mathrm{RO}_{2} \bullet\right)$. The branching of the chain processes involving hydroperoxides suppresses the route of attachment of alkyl and peroxide radicals to the carbon cage structure of the nanotubes and the reaction proceeds in an autocatalytic mode. Contradictory conclusions regarding the effect of CNTs on the chain processes in the oxidation of organic substances (hydrocarbons, polymers) that exist in the literature are attributed to the lack of control over the presence and nature of the metal containing impurities in the CNTs.
\end{abstract}

\section{Introduction}

Carbon nanotubes (CNTs) are a relatively new class of materials, with a broad field of applications. In chemical processes CNTs can be effectively used in the reactions of cycloaddition, epoxidation, cross-combination, photocatalytic and electrochemical reactions. In these processes, CNTs act as carriers of the active phase of catalysts, where metal particles (Fe, Ni, Co, Pd, Pt, Ag, Au, etc.) and/or their oxides are either deposited on the surface of nanotubes or encapsulated inside their cavities [1-6].
Our research has focused on the behaviour of CNTs in the reactions of liquid-phase aerobic oxidation of hydrocarbons. We have previously shown that multi-walled carbon nanotubes (MWCNTs) containing metal (in our case the metals are, as a rule, residues of the catalyst synthesis) actively catalyse the aerobic oxidation of cumene [7] decalin [8] and hydrocarbons in the oil fraction $[9,10]$.

MWCNTs have also been used in the catalytic liquid-phase oxidation of cumene with atmospheric oxygen in the presence of MWCNTs modified on the surface with silver nanocrystals by other authors [11]. The nanotubes were also used in the catalytic oxidation of phenol, nitrobenzene and aniline [12-14]. The field also contains reported aero-

\footnotetext{
* Corresponding author.

Email addresses: zeynalov_2000@yahoo.com (E. Zeynalov); norman_allen@sky.com (N.S. Allen); nazilya.salmanova@gmail.com (N. Salmanova); v.vishnyakov@hud.ac.uk (V. Vishnyakov)
} 
bic selective liquid-phase oxidation of ethylbenzene to acetophenone, where the catalyst was a CNT filled with iron filaments (Fe @ CNTs) [15].

Significantly, in all the above processes, MWCNTs were acting as heterogeneous catalysts and can be used up to 5-6 times without any significant loss of catalytic activity.

Despite these investigations, the question of the mechanism of the catalytic action of CNTs themselves in oxidation processes is still not clear. In principle, the pure carbon framework of a CNT has a fairly high electron affinity (at about $3 \mathrm{eV}$ ) and is capable of actively attaching free radicals [16-19]. As the result of this activity, it has been repeatedly shown that CNTs inhibit chain oxidation processes by actively breaking off the oxidation chains with the formation of inert spin adducts [20-30]. It should be noted, that the antioxidant activity of CNTs was shown mainly for polymeric materials. At the same time, in a number of papers it was indicated that CNTs, on the contrary, possess catalytic activity and accelerate the processes of oxidation of hydrocarbons.

In one example, an industrially oriented process (Shen-zhen Nanotech Port Co. Ltd) nanotubes ware used for the oxidation of cumene [31]. The CNTs material was preliminarily purified with hydrochloric acid, then washed with deionised water to $\mathrm{pH}=6-7$ and dried with hot air at $110^{\circ} \mathrm{C}$ for $24 \mathrm{~h}$. The use of such MWCNTs for the oxidation of cumene at $80^{\circ} \mathrm{C}$, an oxygen flow rate of $10 \mathrm{ml} / \mathrm{min}$ and the amount of MWCNTs added $10 \mathrm{~g} / \mathrm{l}$ resulted in a hydrocarbon conversion of $24.1 \%$ and a selectivity of formation of cumene hydroperoxide of $88.4 \%$. The addition of an acceptor of free radicals (namely, $p$-benzoquinone) to the reaction system almost completely retarded the oxidation process. The authors associate the catalytic activity of the MWCNTs with the initial formation of a complex of CNTs $\mathrm{O}_{2}$ and further development of the reaction by a radical mechanism according to the following scheme:

\section{Scheme 1}

Chain origination

1. CNTs $+\mathrm{O}_{2} \rightarrow$ CNTs $\ldots \mathrm{O}_{2}$

2. CNTs ... $\mathrm{O}_{2}+\mathrm{RH} \rightarrow$ CNTs ... OH $+\mathrm{R} \bullet$

CNTs

3. $\mathrm{ROOH} \rightarrow \mathrm{RO} \bullet+\mathrm{HO} \bullet$

4. $\mathrm{RO} \bullet+\mathrm{RH} \rightarrow \mathrm{ROH}+\mathrm{R} \bullet$

Chain propagation

5. $\mathrm{R} \bullet+\mathrm{O}_{2} \rightarrow \mathrm{ROO} \bullet$

6. $\mathrm{ROO} \bullet+\mathrm{RH} \rightarrow \mathrm{ROOH}+\mathrm{R} \bullet$

7. For Cumene: $\left(\mathrm{C}_{6} \mathrm{H}_{5}\right) \mathrm{CO}_{2} \cdot\left(\mathrm{CH}_{3}\right)_{2} \rightarrow\left(\mathrm{C}_{6} \mathrm{C}_{5}\right) \mathrm{COCH}_{3}+\mathrm{CH}_{3} \bullet$

Where: $\mathrm{RH}$ is a hydrocarbon; $\mathrm{R} \bullet, \mathrm{RO} \bullet, \mathrm{ROO} \bullet$ is an alkyl, alkoxy and peroxide radical, respectively; $\mathrm{ROOH}$ is a hydroperoxide, $\mathrm{ROH}$ is an alcohol.

In another study [32], the authors describe the aerobic liquid-phase oxidation of ethylbenzene in the presence of CNTs obtained and processed similarly to those in the previously mentioned work [31]. In this case, a significant catalytic effect was observed from the use of CNTs. Here, the authors of the study conclude that the presence of CNTs makes it possible to oxidise ethylbenzene to acetophenone in the liquid phase with high selectivity. Unlike cumene oxidation, much more strict conditions of the process were used in this work - the reaction was carried out in a stainless-steel autoclave, in a solvent medium $\left(\mathrm{CH}_{3} \mathrm{CN}\right)$ at a temperature of $155^{\circ} \mathrm{C}$ and an oxygen pressure of $1.5 \mathrm{MPa}$, for $4 \mathrm{~h}$ with a concentration of CNTs to ethylbenzene of $20 \mathrm{~g} / 1$. Under these conditions, the ethylbenzene conversion was $35.6 \%$ and the acetophenone selectivity was $61.2 \%$. It is interesting to note that for an un-purified CNTs sample, the conversion and selectivity indicators were found to be almost the same -38.2 and $60.9 \%$, respectively.
This shows that the CNTs purifying procedure with hydrochloric acid, undertaken to remove metallic impurities, has very little effect on the CNTs activity. As in the case of the oxidation of cumene, the addition of $p$-benzoquinone almost completely inhibits the oxidation of the ethylbenzene. This indicates the radical path of the process. The authors believe that the catalytic activity of CNTs is related to their ability to decompose phenylethyl hydroperoxide into free radicals and to promote the formation of acetophenone through $\pi-\pi$ interactions between radical particles, peroxide and the graphene surface of CNTs. These new catalysts are offered as an effective alternative to cobalt-containing catalytic systems for the selective oxidation of ethylbenzene to acetophenone.

There is published work on the liquid-phase catalytic oxidation of cyclohexane in the presence of CNTs. It is shown that CNTs actively catalyse the oxidation of cyclohexane to cyclohexanone, cyclohexanol, and adipic acid. In this process it is possible to easily manipulate the selectivity of the formation of target products [33].

As an important step in creating a scientific basis for the use of catalytic systems based on nanocarbon structures, the authors of [34] considered the oxidation of cyclohexane in the presence of MWCNTs obtained by the pyrolysis of liquefied petroleum gas on a $\mathrm{Fe}-\mathrm{Mo} / \mathrm{Al}_{2} \mathrm{O}_{3}$ catalyst at $700^{\circ} \mathrm{C}$. Here they utilised single-walled CNTs (SWCNTs) obtained by the thermal-catalytic (Co, Mo) pyrolysis of ethanol on a quartz substrate at $800^{\circ} \mathrm{C}$. It should be noted that un-purified CNTs samples were used in the reactions. Cyclohexane was oxidized in an autoclave with a stirring speed of $1500 \mathrm{rpm}$, a temperature of $125^{\circ} \mathrm{C}$, an oxygen pressure of $1.5 \mathrm{MPa}$ and the presence of a solvent (acetone). A reference compound (butanone) and CNTs in the amount of $0.5 \mathrm{~g} / \mathrm{l}$ of hydrocarbon were also used. The authors of this study, using in situ spectral analysis and kinetic calculation methods according to the density functional theory, put forward a concept explaining the mechanism of the catalytic action of CNTs. They proposed that the reactions occur at the "liquid - surface of CNTs" interface and proceed incomparably more intensely than in the cell of the solvent. In this case, the radicals leading to the oxidation chain accumulate on the graphene layer of the CNTs and stimulate the flow of reactions with electron transfer, resulting in the production of an alcohol and a ketone.

Carbon nanotubes also exhibit high catalytic activity and selectivity in the oxidation of $\alpha$-pinene with a molecular acid [35]. In general, the oxidation of this bicyclic monoterpene proceeds, as a rule, in two main directions - epoxy-degradation and allylic oxidation. The presence of CNTs catalyses the first route, shifting the balance of the target products towards the formation of epoxy compounds. The activity of CNTs as a catalyst is considered by the authors as quite comparable with the activity of metal analogues. Based on the results of these studies, it is argued that catalytic systems based on CNTs are a good alternative to the well-known traditional oxidation catalysts based on metal-containing compounds [36,37]. Nevertheless, in evaluating the outcomes of the above articles [31-37], it is evident that none of them investigated the nature and content of metallic impurities originating from CNTs synthesis. Metal analysis has not been undertaken despite the fact that all the CNTs samples were obtained by the CVD method in the presence of various metal catalysts. In fact, the metal containing impurities, in the form of certain compounds, are practically an integral part of the raw CNTs samples. The subsequent processing of CNTs with mineral acids does not have a particular effect on the activity of the CNTs, and in some cases, the authors simply use un-purified samples of CNTs without any control and allowance of the metal impurity influence on the oxidation processes.

To clarify this issue, we have undertaken a study on the oxidation of cumene in the presence of CNTs obtained by thermal catalytic pyrolysis of cyclohexane. A simple kinetic approach was used, which made it possible to determine the effect of CNTs impurities. 


\section{Experimental}

\subsection{Chemicals and CNT preparation}

In this work the following practical facilities and compounds were used:

1. Hydrocarbon - cumene (Alfa Aesar), purity of the reagent was $99 \%$

2. Radical initiator - azobisisobutyronitrile (AIBN)

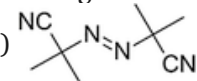

3. Multi-walled carbon nanotubes (MWCNTs) were obtained by the CVD method during the thermal catalytic pyrolysis of cyclohexane in the presence of ferrocene. In the experiments, both, un-purified nanotubes (MWCNTs-c.) and purified nanotubes (treated for $12 \mathrm{~h}$ with hot concentrated hydrochloric acid in a flask with a reflux condenser (MWCNTs-pr.)) were used.

\subsection{Oxygen adsorption technique (cumene)}

The reaction of cumene oxidation was carried out in a reaction vessel with a volume of $30-40 \mathrm{ml}$. The vessel had the form of a closed cylinder with an exit through a thin section into the gasometric installation system, as shown in Fig. 1. The reaction rate was measured by the amount of absorbed oxygen in the air.

For the experiment, the reaction vessel was immersed in an ultra-thermostat and vibrated with a frequency of about $3-7 \mathrm{~Hz}$ to ensure saturation of the reaction mixture with oxygen. The reaction was carried out at a temperature of $333 \mathrm{~K}$. 2-3 min after the vessel was immersed in the thermostat and the vessel was heated, the level of liquid in the burette was combined with the level in the tank and the measurements of the amount of absorbed oxygen were started. With an overpressure in the burette, when the gas evolution is observed, the three-way valve was again returned to its original position, the pressure was released, the liquid levels were combined, and the gas volume measurement procedure was repeated again. Distilled water was used as a thermostatic liquid for a burette and the temperature was $25^{\circ} \mathrm{C}$. The thermostat accuracy of the reaction vessel and burette was $\pm 0.05^{\circ} \mathrm{C}$.

The rate at which a fluid meniscus moves in the burette is proportional to the reaction rate. Oxygen absorption rate of $1 \mathrm{~mm}^{3} / \mathrm{min}$. is:

$1 \mathrm{~mm}^{3} / \min =6.810^{-7} / \mathrm{V}, \mathrm{mol} \mathrm{O}_{2} / 1 \cdot \mathrm{s}$,

where $\mathrm{V}$ is the volume of the reaction mixture in $\mathrm{ml}$.

For the volume of the reaction mixture $10 \mathrm{ml}$, we have $1 \mathrm{~mm}^{3} / \mathrm{min}=6.81 \times 10^{-8} \mathrm{~mol} \mathrm{O}_{2} / 1 \cdot \mathrm{s}$. The oxidation rate was determined from the tangent of the slope of the kinetic dependences of oxy-

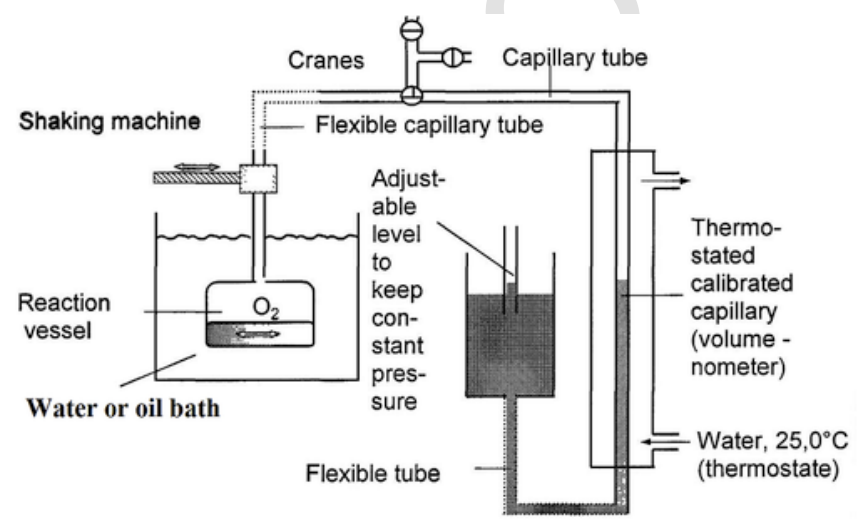

Fig. 1. Schematic representation of the laboratory setup for measuring the amount of gas absorbed or released [38-41]. gen absorption: $\mathrm{dO}_{2} / \mathrm{dt}=\mathrm{WO}_{2}$. Each experiment was repeated three times, the error in determining the reaction rate was around $3-7 \%$.

\subsection{Mult-walled CNT's}

The synthesis of MWCNTs was carried out in the laboratory of Scientific Instruments in Dresden GMBH, SCIDRE by pyrolysis of cyclohexane (CG) in the presence of ferrocene (FC) as a catalyst at $900^{\circ} \mathrm{C}$ for $1 \mathrm{~h}$, followed by chemical precipitation of the carbon from the gas phase. Synthesis precursors, taken in the FC/CG ratio $=20 \mathrm{mg} / \mathrm{ml}$, were supplied to the reaction zone as an aerosol-assisted (AACVD) process [42].

The structure and composition of the MWCNTs was analysed by scanning electron microscopy (SEM) and X-ray diffraction analysis (XRD).

The outer diameter of the nanotubes was, on average, $30-40 \mathrm{~nm}$ (Fig. 2).

At this stage our analytical assessment is based on a not so fully successive CNT purification and the evidence of metallic impurities as indicated by XRD. More detailed investigations into CNT's are in processes including variable preparative methodologies and the results will be published with detailed TEM and XPS analysis.

\section{Results and discussion}

The kinetic curves of oxygen absorption in the initiated oxidation of cumene in the presence of untreated and purified MWCNTs samples are shown in Fig. 3.

As can be seen from the kinetics, the oxidation reaction of cumene in the presence of as synthesised MWCNTs-c. is inhibited at the initial stages and proceeds with a certain induction period [Fig. 3 (2)]. After exiting the induction period, the reaction is catalysed and proceeds at rates exceeding the rate initiated by the oxidation of the control reaction [Fig. 3 (kinetics 1)]. Reactions with purified samples (MWCNT-pr.) proceed without any induction period and are actively catalysed by the addition of the nanotubes [Fig. 3 (kinetics 3-5)].

Fig. 4A and B shows the data for the XRD analysis of the MWCNTs-c. samples and MWCNTs-in its purified form.

From the XRD data it can be seen that in the as synthesised samples the crystalline phase of iron carbide $\mathrm{Fe}_{7} \mathrm{C}_{3}$ and iron in its $\alpha$-form is present in significant amount. The treatment of MWCNTs with hot hydrochloric acid only leads to a different packing of the metal atoms in the crystal lattice with the formation of $\mathrm{Fe}_{3} \mathrm{C}$ cohenite and $\gamma$-form iron. Thus, the purification of CNTs by treatment with hydrochloric acid, which is usually used for the purification of CNTs from impurities of iron-containing catalysts, does not have the desired purification effect. Iron and iron-containing compounds continue to be part of the MWCNTs and actively catalyse the oxidation of hydrocarbons, interacting with the hydroperoxides according to the well-known Haber - Weiss scheme, providing degenerate branching of the chains, accompanied by the formation of active radicals $[43,44]$.

It is evident from the kinetic analysis of the oxidation of the hydrocarbons in the presence of MWCNTs-c. The initial inhibition process is observed as a consequence of the interaction of the carbon skeleton with free radicals up to a point where a critical concentration of hydroperoxides accumulates. Furthermore, the radical decomposition of the hydroperoxides, as catalysed by the iron compounds in MWCNTs-c., completely inhibits the antioxidant component of the process and the reaction proceeds in an autocatalytic mode. In the case of the use of MWCNTs-pr. samples, the catalytic action of $\mathrm{Fe}_{3} \mathrm{C}$ and $\gamma$-Fe plateaus the induction period at the onset of the reaction.

From the analysis of the literature and experimental data considered here, it can be concluded that CNTs free from any impurities can actively accept free alkyl $\mathrm{R} \bullet$ radicals and considerably less active per- 


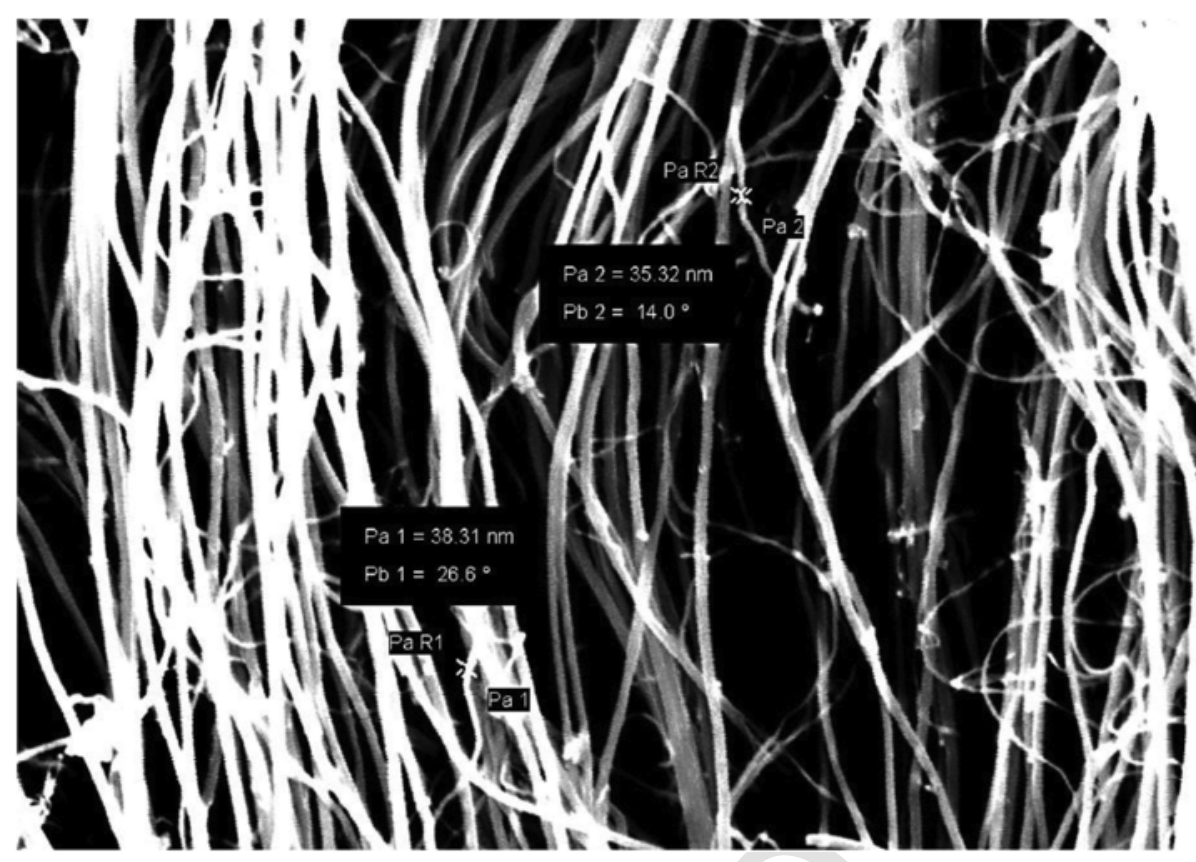

Fig. 2. A snapshot of the MWCNTs taken with a scanning electron microscope.

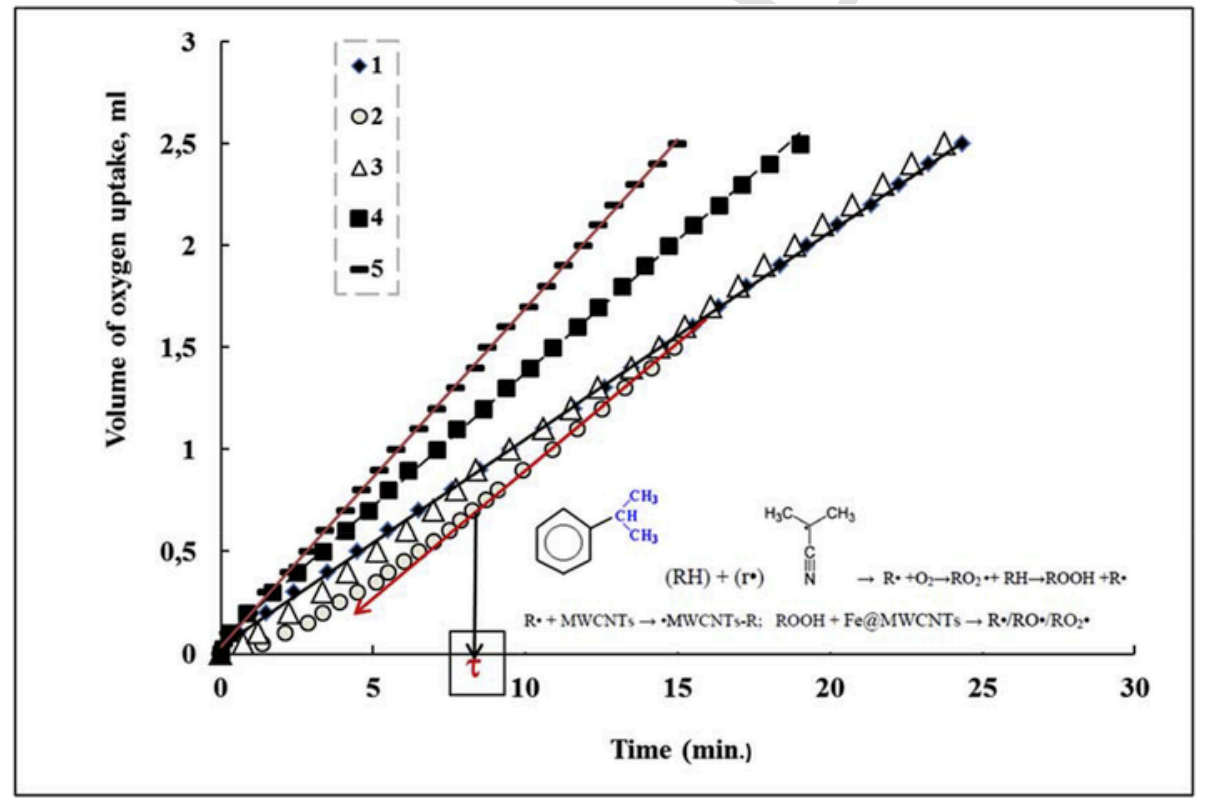

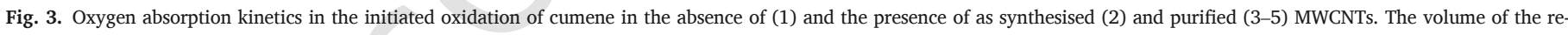

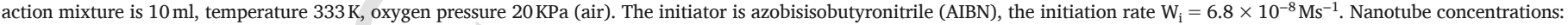
[MWCNTs]: 1-0; 2 - 2g/l; (MWCNT-c.); 3-0.5g/1, 4 - 1 g/1, 5 - 2g/l (MWCNTs-pr.).

oxide $\mathrm{RO}_{2} \bullet$ radicals $[18,45,46]$. This conclusion is in good agreement with the data on other nanocarbon compounds - fullerenes $\mathrm{C}_{60}$ and $\mathrm{C}_{70}$ and graphene [23,26,47-53]. Graphene, as is known, has an electron affinity of $4.24 \mathrm{eV}[54,55]$, which is much higher than the similar value for fullerenes and CNTs, and therefore, even when graphene is doped with variable valence metal salts, its catalytic properties are poor. For comparison, the oxidation reaction of naphthenic hydrocarbons from the diesel fraction $216-360{ }^{\circ} \mathrm{C}$ at $135-140{ }^{\circ} \mathrm{C}$ in a bubble reactor in the presence of the catalytic system MWCNTs + Mn naphthenate (0.1-0.2 wt.\% for raw materials) leads to a record-high yield of synthetic naphthenic acids (SNA) $\geq 23 \%$. On the other-hand, the use of graphene in identical conditions gives a yield of SNA of not more than $17 \%$ [9,56-60]. This conclusion is also supported by the fact that the antioxidant activity of CNTs is well fixed mainly during thermo-and photo-oxidative aging of various polymer compositions, that is, under conditions of diffusional restrictions for oxygen access [61-64]. Under these conditions, the macro radicals are relatively long-lived. However, in the liquid phase with a sufficiently high oxygen pressure of $13.3-26.7 \mathrm{kPa}$, when the concentration of oxygen dissolved in a hydrocarbon is of the order of $\sim 10^{-3} \mathrm{M}$, the alkyl radicals $\mathrm{R} \bullet$ almost instantaneously change into a peroxide by the reaction $\mathrm{R} \bullet+\mathrm{O}_{2}$ $\rightarrow \mathrm{RO}_{2} \bullet[65,66]$. At this stage, linear breakage of radicals on the carbon nanotubes almost disappears or proceeds at low rates. Thus, in the presence of metals in the composition of the CNTs, the reaction of CNTs $+\mathrm{R} \bullet\left(\mathrm{RO}_{2} \bullet\right) \rightarrow \bullet$ CNTs-R $\left(\mathrm{RO}_{2}{ }^{\circ}\right)$ cannot compete with the reaction $\mathrm{ROOH}+\mathrm{M} @ \mathrm{CNTs} \rightarrow$ radicals $\mathrm{RO} \bullet\left(\mathrm{RO}_{2} \bullet\right)$ and the oxidation process develops into a cat- 
$\mathbf{A}$

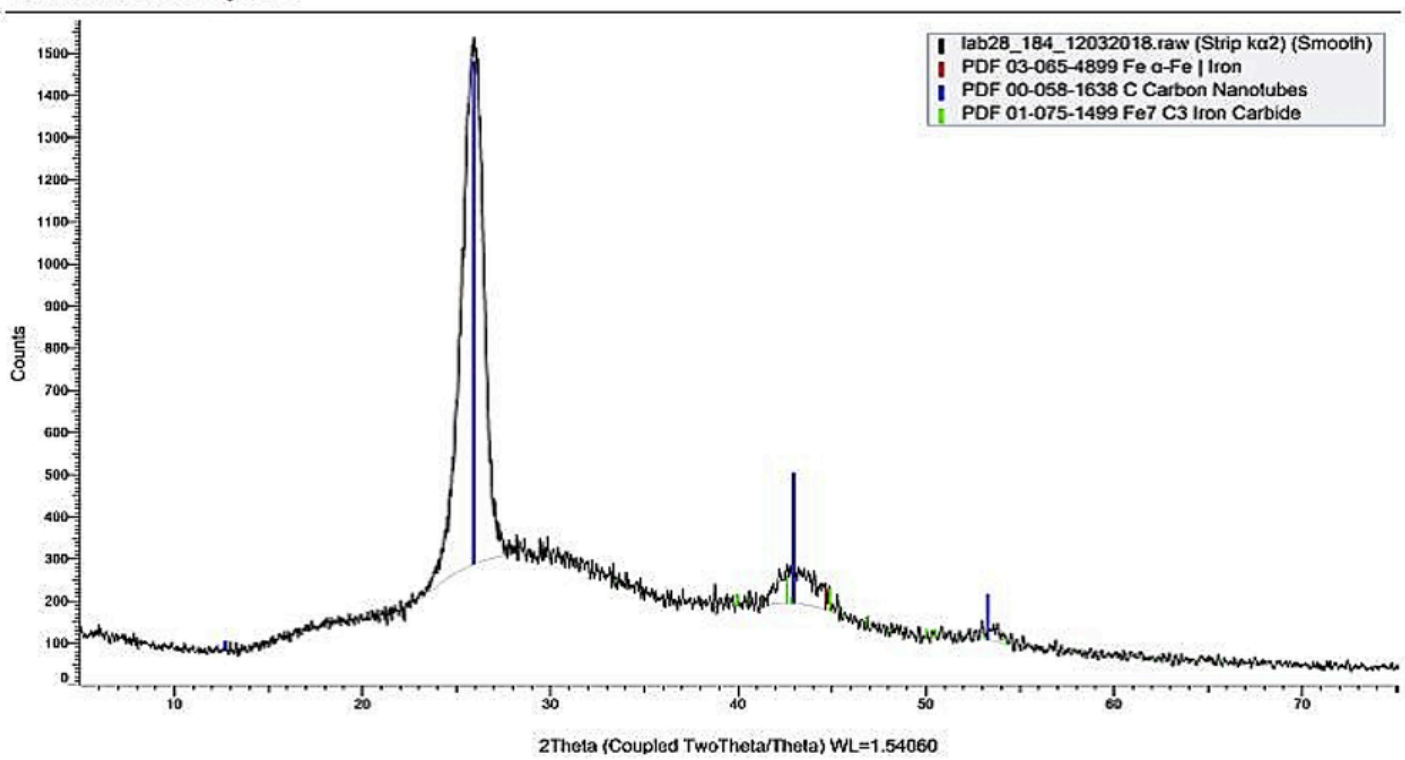

Commander Sample ID

B

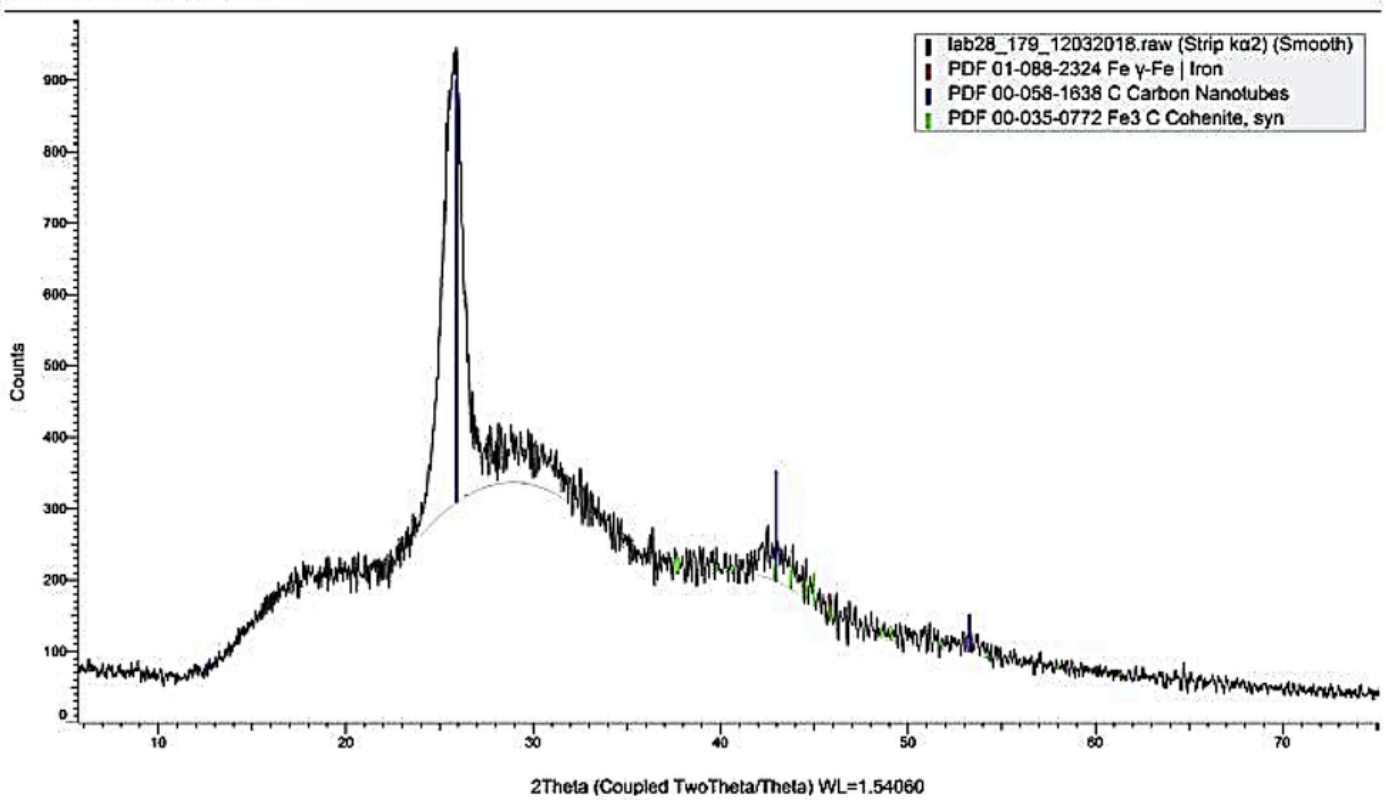

Fig. 4. XRD from as synthesised (A) and hydrochloride acid-purified (B) samples of multi-walled carbon nanotubes.

alytic mode.

Scheme 2

Chain origination:

1. AIBN $\rightarrow \perp$; $\left(\mathrm{T}^{\circ} \mathrm{C}\right) \mathrm{R} \bullet(\mathrm{RO} 2 \bullet)+\mathrm{RH} \rightarrow \mathrm{RH}+\mathrm{R} \bullet\left(\mathrm{W}_{\mathrm{i}}\right.$ initiation rate $)$

Propagation of the chain:

2. $\mathrm{R} \bullet+\mathrm{O}_{2} \rightarrow \mathrm{RO}_{2} \bullet$

3. $\mathrm{RO}_{2} \bullet+\mathrm{RH} \rightarrow \mathrm{ROOH}+\mathrm{R} \bullet$

Degenerate chain branching:

4. $\mathrm{ROOH}+\mathrm{Fe} @ \mathrm{MWCNTs} \rightarrow \mathrm{RO} \bullet / \mathrm{RO}_{2} \bullet$

Chain termination:

5. R• + MWCNTs $\rightarrow \bullet$ MWCNT-R

6. $\mathrm{RO}_{2} \bullet+\mathrm{RO}_{2} \square \rightarrow$ molecular products where $\mathrm{R} \bullet\left(\mathrm{RO}_{2} \bullet\right)$ are the radicals formed during the disintegration of the initiator, the other designations are indicated in Scheme 1.

* The conventional numbering of oxidation stages has been used $[67,68]$.

\section{Conclusions}

1. Carbon nanotubes, obtained by chemical vapour deposition during the thermal catalytic pyrolysis of hydrocarbons in the presence of variable valence metal compounds, contain, as a rule, catalyst residues (impurities) - metals and metal carbides. The impurities catalyse the processes of chain liquid-phase aerobic oxidation of hydrocarbons by the radical decomposition of hydroperoxides. 
2. The effect of MWCNTs obtained by the thermal catalytic pyrolysis of cyclohexane in the presence of ferrocene as a catalyst on the kinetics of the oxidation processes (azobisisobutyronitrile) of cumene by atmospheric oxygen has been investigated. It has been established that the degenerate branching of the chain oxidation process suppresses the route of attachment of alkyl and peroxide radicals to the carbon cage of nanotubes inherent in pure CNTs, and the reaction proceeds in an autocatalytic mode.

3. Using the example of cleaning CNTs containing $\mathrm{Fe}_{7} \mathrm{C}_{3}$ and iron in the $\alpha$-form, it was found that cleaning CNTs from metal carbides by treatment with hydrochloric acid leads only to a different packing of the metal atoms in the crystal lattice $\left(\mathrm{Fe}_{3} \mathrm{C}\right.$ and $\gamma$-iron).

4. A clearer and more effective reaction scheme for understanding the oxidation of hydrocarbons in the presence of CNTs as obtained by the CVD gas phase method during the thermal-catalytic pyrolysis of carbon-containing raw materials has been proposed.

\section{References}

[1] S.A. Miners, G.A. Rance, A.N. Khlobystov, Chemical reactions confined within carbon nanotubes, Chem. Soc. Rev. 45 (17) (2016) 4727-4746.

[2] A. La Torre, G.A. Rance, J. El Harfi, J. Li, D.J. Irvine, P.D. Brown, A.N. Khlobystov, Transport and encapsulation of gold nanoparticles in carbon nanotubes, Nanoscale 2 (6) (2010) 1006-1010.

[3] G.A. Rance, W.A. Solomonsz, A.N. Khlobystov, Click chemistry in carbon nanoreac tors, Chem. Commun. 49 (11) (2013) 1067-1069.

[4] G.A. Rance, D.H. Marsh, S.J. Bourne, T.J. Reade, A.N. Khlobystov, Van der Waals interactions between nanotubes and nanoparticles for controlled assembly of composite nanostructures, ACS Nano 4 (8) (2010) 4920-4928.

[5] B. Cornelio, G.A. Rance, M. Laronze-Cochard, A. Fontana, J. Sapi, A.N. Khlobystov Palladium nanoparticles on carbon nanotubes as catalysts of cross-coupling reactions, J. Mater. Chem. 1 (31) (2013) 8737-8744.

[6] B. Cornelio, A.R. Saunders, W.A. Solomonsz, M. Laronze-Cochard, A. Fontana, J. Sapi, G.A. Rance, Palladium nanoparticles in catalytic carbon nanoreactors: the effect of confinement on Suzuki-Miyaura reactions, J. Mater. Chem. 3 (7) (2015) 391.

[7] Yu.P. Cherepnova, E.B. Zeynalov, N.Ya. Ishenko, M.M. Abdullayev, Influence of the metal-containing water-soluble multi-walled carbon nanotubes on reaction of a model hydrocarbon oxidation, Oil Gas Eng. 6 (2013) 66-69.

[8] E.B. Zeynalov, N.Ya. Ishenko, M.Ya. Magerramova, N.I. Salmanova, M.M. Abdullayev, Liquid-phase aerobic oxidation of decalin in the presence of metal-containin multi-layered carbon nanotubes, Oil Gas Eng. 2 (2016) 73-76.

[9] E.B. Zeynalov, A.Z. Aliyeva, L.G. Nuriyev, H.J. Ibragimov, N.Ya. Ishenko, Mult walled carbon nanotubes containing metal as a catalyst, Oil Gas Eng. 6 (2011) 69-72.

[10] E.B. Zeynalov, G.G. Nasibova, Peculiarities of the aerobic oxidation of oil hydrocarbons catalyzed by carbon nanocompounds, Rep. Azerbaijan Natl. Acad. Sci. LXXI (1) (2016) 44-48.

[11] N.S. Kobotaeva, T.S. Skorokhodova, N.V. Ryabova, Catalytic systems of cumene oxidation based on multiwalled carbon nanotubes, Russ. J. Phys. Chem. 89 (3) (2015) 462-468.

[12] S. Yang, X. Li, W. Zhu, J. Wang, C. Descorme, Catalytic activity, stability and structure of multi-walled carbon nanotubes in the wet air oxidation of phenol, Carbon 46 (3) (2008) 445-452.

[13] S. Yang, W. Zhu, X. Li, J. Wang, Y. Zhou, Multi-walled carbon nanotubes (MWNTs) as an efficient catalyst for catalytic wet air oxidation of phenol, Catal. Commun. 8 (12) (2007) 2059-2063.

[14] S. Yang, Y. Sun, H. Yang, J. Wan, Catalytic wet air oxidation of phenol, nitrobenzene and aniline over the multi-walled carbon nanotubes (MWCNTs) as catalysts, Front. Environ. Sci. Eng. 9 (3) (2015) 436-443.

[15] J. Luo, H. Yu, H. Wang, F. Peng, Enhancing the catalytic activity of carbon nanotubes by filled iron nanowires for selective oxidation of ethylbenzene, Catal. Commun. 51 (2014) 77-81.

[16] F. Buonocore, F. Trani, D. Ninno, A. Di Matteo, G. Cantele, G. Iadonisi, Ab initio calculations of electron affinity and ionization potential of carbon nanotubes, Nanotechnology 19 (2) (2007), 025711.

[17] I. Fenoglio, M. Tomatis, D. Lison, J. Muller, A. Fonseca, J.B. Nagy, B. Fubini, Reac tivity of carbon nanotubes: free radical generation or scavenging activity?, Free Radic. Biol. Med. 40 (7) (2006) 1227-1233.

[18] A. Galano, Carbon nanotubes: promising agents against free radicals, Nanoscale 2 (3) (2010) 373-380.

[19] A. Martínez, A. Galano, Free radical scavenging activity of ultrashort single-walled carbon nanotubes with different structures through electron transfer reactions, J. Phys. Chem. C 114 (18) (2010) 8184-8191.

[20] P.C.P. Watts, P.K. Fearon, W.K. Hsu, N.C. Billingham, H.W. Kroto, D.R.M. Walton, Carbon nanotubes as polymer antioxidants, J. Mater. Chem. 13 (3) (2003) 491-495.

[21] M.J. Martínez-Morlanes, P. Castell, P.J. Alonso, M.T. Martinez, J.A. Puértolas, Multi-walled carbon nanotubes acting as free radical scavengers in gamma-irradiated ultrahigh molecular weight polyethylene composites, Carbon 50 (7) (2012) 2442-2452.
[22] X. Shi, B. Jiang, J. Wang, Y. Yang, Influence of wall number and surface functionalization of carbon nanotubes on their antioxidant behavior in high density polyethylene, Carbon 50 (3) (2012) 1005-1013.

[23] E.B. Zeynalov, J.F. Friedrich, Anti-radical activity of fullerenes and carbon nanotubes in reactions of radical polymerization and polymer thermal/thermo-oxidative degradation: a review, Mater. Test. 49 (5) (2007) 265-270.

[24] E.B. Zeynalov, J.F. Friedrich, Antioxidative activity of carbon nanotube and nanofiber, Open Mater. Sci. J. 2 (2008) 28-34.

[25] L. Guadagno, C. Naddeo, M. Raimondo, G. Gorrasi, V. Vittoria, Effect of carbon nanotubes on the photo-oxidative durability of syndiotactic polypropylene, Polym. Degrad. Stabil. 95 (9) (2010) 1614-1626.

[26] E. Zeynalov, T. Nagiyev, J. Friedrich, M. Magerramova, Carbonaceous nanostructures in hydrocarbons and polymeric aerobic oxidation mediums, in: Alexander Mihai Grumezescu (Ed.), The Book: Fullerenes, Graphenes and Nanotubes: a Pharmaceutical Approach, Elsevier -William Andrew Publishing House, 2018, pp. 631-681, chapter 16.

[27] S. Bocchini, A. Frache, G. Camino, M. Claes, Polyethylene thermal oxidative stabilisation in carbon nanotubes based nanocomposites, Eur. Polym. J. 43 (8) (2007) 3222-3235.

[28] P.R. Sreekanth, N.N. Kumar, S. Kanagaraj, Improving post irradiation stability of high-density polyethylene by multi walled carbon nanotubes, Compos. Sci. Technol. 72 (3) (2012) 390-396.

[29] S. Morlat-Therias, E. Fanton, J.L. Gardette, S. Peeterbroeck, M. Alexandre, P. Dubois, Polymer/carbon nanotube nanocomposites: influence of carbon nanotubes on EVA photodegradation, Polym. Degrad. Stabil. 92 (10) (2007) 1873-1882.

[30] Z. Shen, S. Bateman, D.Y. Wu, P. McMahon, M. Dell'Olio, J. Gotama, The effects of carbon nanotubes on mechanical and thermal properties of woven glass fibre reinforced polyamide-6 nanocomposites, Compos. Sci. Technol. 69 (2) (2009) 239-244.

[31] S. Liao, F. Peng, H. Yu, H. Wang, Carbon nanotubes as catalyst for the aerobic oxidation of cumene to cumene hydroperoxide, Appl. Catal. Gen. 478 (2014) 1-8.

[32] J. Luo, F. Peng, H. Yu, H. Wang, W. Zheng, Aerobic liquid-phase oxidation of ethylbenzene to acetophenone catalyzed by carbon nanotubes, ChemCatChem 5 (6) (2013) 1578-1586.

[33] H. Yu, F. Peng, J. Tan, X. Hu, H. Wang, J. Yang, W. Zheng, Selective catalysis of the aerobic oxidation of cyclohexane in the liquid phase by carbon nanotubes, Angew. Chem. 123 (17) (2011) 4064-4068.

[34] X. Yang, H. Wang, J. Li, W. Zheng, R. Xiang, Z. Tang, H. Yu, F. Peng, Mechanistic insight into the catalytic oxidation of cyclohexane over carbon nanotubes: kinetic and in situ spectroscopic evidence, Chem. Eur. J. 19 (30) (2013) 9818-9824.

[35] Y. Cao, Y. Li, H. Yu, F. Peng, H. Wang, Aerobic oxidation of $\alpha$-pinene catalyzed by carbon nanotubes, Catal. Sci. Technol. 5 (8) (2015) 3935-3944.

[36] Y. Zhai, Z. Zhu, S. Dong, Carbon-based nanostructures for advanced catalysis, ChemCatChem 7 (18) (2015) 2806-2815.

[37] X. Sun, R. Wang, D. Su, Research progress in metal-free carbon-based catalysts, Chin. J. Catal. 34 (2013) 508-523.

[38] N.M. Emanuel, G.P. Gladyshev, V.F. Tsepalov, K.B. Piotrovskiy, Testing of Chemica Compounds as Stabilizers for Polymer Materials (Preprint). Chernogolovka (Russ.), Institute of Chemical Physics of the USSR Academy of Sciences, 1973, 52pp.

[39] G.P. Gladyshev, V.F. Tsepalov, Testing of chemical compounds as stabilizers for polymer materials, Russ. Chem. Rev. 44 (10) (1975) 1830-1850.

[40] E.B. Zeynalov, O.A. Vasnetsova, Kinetic Screening of Inhibitors of Radical Reac tions, Elm, Baku, 1993, 228pp.

[41] E.B. Zeynalov, N.S. Allen, Simultaneous determination of the content and activity of sterically hindered phenolic and amine stabilizers by means of an oxidative model reaction, Polym. Degrad. Stabil. 85 (2) (2004) 847-853.

[42] S.H. Abdullayeva, N.N. Musayeva, C. Frigeri, A.B. Huseynov, R.B. Jabbarov, R.B. Abdullayev, Ch.A. Sultanov, R.F. Hasanov, Characterization of high-quality carbon nanotubes synthesized via Aerosol-CVD, J. Adv. Phys. 11 (3) (2015) 3229-3240.

[43] N.M. Emanuel, Z.K. Maizus, I.P. Skibida, The catalytic activity of transition metal compounds in the liquid-phase oxidation of hydrocarbons, Angew Chem. Int. Ed. Engl. 8 (2) (1969) 97-107.

[44] V.M. Potekhin, Basic Foundation of the Theory of Chemical Processes of Technology of Organic Substances and Oil Refining, Khimizdat, St. Petersburg (Russ.) 2014, 311pp.

[45] E.B. Zeynalov, J.F. Friedrich, Antioxidant properties of multiwall carbon nanotubes: first measurements using a model oxidative reaction, Polym. Polym. Compos. 14 (8) (2006) 779-785

[46] N.I. Salmanova, M.Ya. Magerramova, M.M. Agahuseynova, E.B. Zeynalov, Thermal analysis of polyethylene composites containing fullerenes and carbon nanotubes, Int. Res. J. Emerg. Trends Multidisciplinary 1 (10) (2015) 220-225.

[47] R.G. Bulgakov, Y.G. Ponomareva, S.I. Maslennikov, E.Y. Nevyadovsky, S.V. Antipina, Inertness of $\mathrm{C} 60$ fullerene toward RO2 peroxy radicals, Russ. Chem. Bull. 54 (8) (2005) 1862-1865

[48] D.I. Galimov, R.G. Bulgakov, D.R. Gazeeva, Reactivity of fullerene C 60 towards peroxy radicals generated by liquid-phase oxidation of cumene and ethylbenzene with oxygen, Russ. Chem. Bull. 60 (10) (2011) 2107-2109.

[49] D.S.H. Sabirov, R.R. Garipova, R.G. Bulgakov, What is fullerene is more reactive towards peroxyl radicals? A comparative DFT Study on ROO • addition to C60 and C70 fullerenes, Fullerenes, Nanotub. Carbon Nanostruct. 23 (12) (2015) 1051-1057.

[50] R.K. Yumagulova, N.A. Medvedeva, L.R. Yakupova, S.V. Kolesov, R.L. Safiullin, Free-radical chain oxidation of 1,4-dioxane and styrene in the presence of fullerene C60, Kinet. Catal. 54 (6) (2013) 709-715.

[51] E.B. Zeynalov, Thermooxidative degradation of the low-density polyethylene in the presence of fullerenes C60/C70, in: Omari Mukbaniani, A. Marc, M. Abadie, Tamar Tatrishvili (Eds.), High-performance Polymers for Engineering-based Composites, 
[52] E.B. Zeynalov, M. Wagner, J. Friedrich, M.Ya. Magerramova, N.I. Salmanova, Antioxidant traits for some carbon moieties integrated in polymer materials (A review), Chem. Chem. Technol. 10 (4s) (2016) 581-586.

[53] E.B. Zeynalov, N.S. Allen, N.I. Salmanova, Radical scavenging efficiency of different fullerenes C60 - C70 and fullerene soot, Polym. Degrad. Stabil. 94 (8) (2009) 1183-1189.

[54] G. Fiori, A. Betti, S. Bruzzone, G. Iannaccone, Lateral graphene - hBCN heterostructures for fully two-dimensional transistors, ACS Nano 6 (3) (2012) 2642-2648.

[55] P. Palla, G.R. Uppu, A.S. Ethiraj, J.P. Raina, Bandgap engineered graphene and hexagonal boron nitride for resonant tunneling diode, Bull. Mater. Sci. 39 (6) (2016) 1441-1451.

[56] A.Z. Aliyeva, L.G. Nuriyev, E.B. Zeynalov, Production of synthetic petroleum acids by catalytic oxidation of naphthenic concentrate with participation of nanocarbon structures, Azerbaijan Oil Econ. 10 (2009) 47-50.

[57] V.M. Abbasov, L.I. Aliyeva, H.M. Alma, L.G. Nuriyev, L.M. Efendiyeva, Liquid-phase oxidation of the oil naphthenic fraction in the presence of nanostructured graphene, In: IX International Scientific Conference "Fullerenes and Nanostructures in Condensed Matter", Scientific Journal, Minsk (Belarus), 2016, pp. 330-335.

[58] L.M. Afandiyeva, V.M. Abbasov, L.I. Aliyeva, L.H. Nuriyev, A.D. Guliyev, H. Alma T. Salan, Synthesis of Petroleum Acids Based on Naphthene-paraffinic Oil Present in the Catalytic Presence of Reduced Graphene Oxide Processes of Petrochemistry and Oil-refining, 2016302-315, 17, 4 (65).

[59] L.I. Aliyeva, L.M. Afandiyeva, V.M. Abbasov, H.J. Ibrahimov, A.D. Guliyev, The process of reducing the amount of the salt and the grains of the condensation of the mineral salts, Process Petrochem. Oil Refin. 18 (3) (2017) 202-214, 65.
[60] L.M. Afandiyeva, V.M. Abbasov, L.I. Aliyeva, L.H. Nuriyev, V.Kh. Musali, Synthesis of petroleum and oxy-acids based on naphthene- paraffinic hydrocarbons in the cat alytic presence of various modification of graphene, In: 3-rd Turkic World Conference on Chemical Sciences and Technologies, 10-13 September 2017, Baku, Azerbaijan, 207 (PO 63).

[61] N.M. Emanuel, V.A. Roginsky, A.L. Buchachenko, Some problems of the kinetics of radical reactions in solid polymers, Adv. Chem. 51 (3) (1982) 361-393.

[62] N.M. Emanuel, A.L. Buchachenko, Chemical physics of molecular destruction and stabilization of polymers, M.: Science (1988), 368pp.

[63] Yu.A. Shlyapnikov, Antioxidant stabilization of polymers, Adv. Chem. 50 (6) (1981) 1105-1140.

[64] E.B. Zeynalov, Anticatalysts of Thermooxidative Degradation of Polymeric Materials. Baku: Elm, 2014, 160pp.

[65] N.M. Emanuel, E.T. Denisov, Z.K. Mayzus, Chain reactions of oxidation of hydrocarbons in the liquid phase, M.: Science (1965) 17.

[66] B. Maillard, K.U. Ingold, J.C. Scaiano, Oxygen in solution, J. Am. Chem. Soc. 105 (15) (1983) 5095-5099.

[67] N.M. Emanuel, E.T. Denisov, Z.K. Mayzus, Chain reactions of oxidation of hydrocarbons in the liquid phase, M.: Science. (1965), 374pp.

[68] G. Scott, Atmospheric Oxidation and Antioxidants, vols. I II and III, Elsevier, London, 1993, pp. 234, 542 and 376. 